\title{
Topographie multi-échelle pour le filtrage optique multicouche large-bande
}

\author{
C. Amra, C. Deumié, J.Y. Natoli et M. Commandré \\ Institut Fresnel Marseille, CNRS/ENSPM, Université d'Aix Marseille, Université de Provence, \\ École Nationale Supérieure de Physique de Marseille, Domaine Universitaire de Saint Jérôme, \\ 13397 Marseille cedex 20, France
}

\begin{abstract}
Résumé : On s'intéresse aux problèmes multi-échelles qui interviennent lorsque l'on aborde la réalisation et la caractérisation de systèmes interférentiels multicouches pour des applications de type filtrage optique allant de I'UV (150 mm) au moyen infra-rouge $(16 \mu \mathrm{m})$. Ces difficultés concernent les propriétés optiques, à caractère radiatif ou dissipatif, mais aussi les propriétés thermiques, mécaniques et climatiques, ou de tenue au flux laser... On montre l'importance de la dispersion spectrale de la rugosité pour un polissage multi-échelle, ou le rôle crucial de nana-centres faiblement distribués et initiateurs des processus d'endommagement laser..
\end{abstract}

\section{INTRODUCTION}

Les systèmes interférentiels multicouches, ou empilements de couches minces optiques, sont largement utilisés pour le filtrage spectral de la lumière, parce qu'ils permettent de modifier pratiquement à volonté les facteurs de réflexion ou de transmission des composants qui interagissent avec la lumière incidente... Pour atteindre une fonction ou un gabarit optique particulier, des méthodes de synthèse sophistiquées doivent souvent être mises en œuvre, et peuvent conduire à des empilements complexes présentant des pertes par absorption, diffusion, luminescence ... On associe donc à ces composants un bilan énergétique plus ou moins détaillé sous la forme : $\quad I=R+T+A+D+\ldots$

Où :

- $\quad R$ et $T$ désignent les facteurs de réflexion et de transmission en intensité ; ces grandeurs suffisent en général si la précision requise sur le bilan n'excède pas le pour-cent

- A désigne la quantité de lumière absorbée, en relation directe avec l'indice imaginaire des matériaux. Elle conduit à une élévation de température du composant, et adresse ainsi les problèmes de thermique photo-induite

- D désigne l'intégrale de la lumière diffusée en dehors des directions spéculaires, qui résulte de la présence de rugosités de surface ou d'hétérogénéités de volume dans les multicouches et substrats. Ces pertes par diffusion limitent en particulier les taux d'isolation dans les éclateurs spectraux etc

De façon générale, pour les applications de l'optique moderne, mettant en jeu une technologie de pointe, on exige un bilan énergétique souvent à mieux que $10^{-4}$, ce qui requiert une maîtrise et compréhension approfondies de ces phénomènes de pertes. Mais au delà du bilan optique, les propriétés mécaniques, climatiques, thermiques, de vieillissement sous flux optique ou particulaire, doivent aussi être maîtrisées. Dans cet exposé nous montrons comment les problèmes multi-échelles interviennent dès lors que l'on veut avancer dans ces investigations.

\section{FABRICATION DE FILTRES OPTIQUES MULTICOUCHES}

Pour les applications optiques, les substrats mis en jeu sont de nature et géométrie diverses. On veut en effet modifier les propriétés des verres, semi-conducteurs, métaux, cristaux, plastiques ou résines..., et les surfaces peuvent être planes ou courbes, avec des rayons de courbure quelquefois très faibles (cas des micro-pointes). De plus, les dimensions à traiter s'étalent de quelques microns (cas des micro-lentilles ou 
des extrémités de fibres optiques, des faces latérales ou verticales de diodes) à quelques mètres (cas des miroirs de télescope), ce qui représente environ six décades sur les diamètres utiles des composants. On imagine d'emblée que les techniques de nettoyage, polissage, caractérisation..., seront très différentes dans le principe, selon que l'on veut traiter un diamètre métrique ou micro-métrique.

On admet également que les techniques de fabrication et croissance puissent être très différentes de celles très connues de la micro-électronique, en particulier parce que les substrats sont amorphes et supportent rarement une température élevée. De plus, pour couvrir un domaine spectral allant de l'UV $(150 \mathrm{~nm})$ au moyen infra-rouge $(16 \mu \mathrm{m})$, il faut savoir déposer en couches minces environ une vingtaine de matériaux, et savoir les associer entre eux, avec des épaisseurs interférentielles, sous n'importe quelle séquence et sur tous types de substrats, ce qui nous éloigne de toute considération sur la compatibilité des mailles... A titre d'exemple, un filtre bande étroite de type télécommunications optiques (dense wavelength multiplexing) requiert le dépôt de près d'une centaine de couches minces optiques, pour atteindre une bande passante d'une fraction de $\mathrm{nm}$, avec de forts taux de réjection et un front rectangulaire.

Les matériaux couramment utilisés sont en général les sulfures, fluorures, nitrures, et les oxydes... Ils peuvent être déposés par les techniques d'évaporation-condensation sous vide (Electron Beam Deposition), qui sont peu coûteuses avec un nombre limité de paramètres à contrôler. Toutefois ces techniques conduisent souvent à des structures lacunaires ou hétérogènes des composants observés à l'échelle microscopique, induisant une fragilité mécanique et des phénomènes de diffusion, d'adsorption avec dérive climatique... Pour pallier ces difficultés, on augmente la mobilité des particules au voisinage des substrats en faisant appel, par exemple, à une assistance par faisceau d'ions (Ion Assisted Deposition) ou plasma (Ion Plating). Pour gagner en pureté ou stabilité de process, on peut aussi tenter de remplacer l'évaporation par de l'ablation ou pulvérisation ionique (Ion Beam Sputtering), mais il existe plusieurs autres méthodes plus ou moins récentes (Plasma Plating, Double Ion Beam Sputtering, Plasma Ion Assisted Deposition, Pulvérisation Cathodique...). Dans tous ces derniers cas, les couches obtenues sont amorphes avec une compacité pratiquement optimale, proche du matériau massif, avec de bonnes propriétés mécaniques et climatiques.

Lorsque le procédé de fabrication est choisi, c'est alors le système de contrôle de formation in situ de l'empilement qui joue un rôle majeur. Si plusieurs techniques (microbalance à quartz...) sont disponibles pour contrôler et réaliser des fonctions optiques relativement « stables » de type antireflet ou miroirs..., il ne fait pas de doute qu'un système de contrôle optique in-situ est incontournable pour réaliser des filtres à bande très étroite (de l'ordre du $\mathrm{nm}$ ou plus), ou pour positionner des dichroíques avec une précision inégalable. En particulier ce type de contrôle permet de bénéficier de la compensation d'erreurs, et de prévenir toute dérive spectrale lors de la mise à l'air du composant. On retiendra que, selon que l'application recherchée est large-bande ou centrée sur quelques raies, le contrôle optique est indispensable ou optionnel.

On conclura cette partie en soulignant le fait que le rapport des longueurs d'onde extrême (150 nm$16 \mu \mathrm{m}$ ), pour nos domaines d'application, n'est pas loin de deux décades: $\lambda^{\prime} / \lambda \approx 100$. Dans ces conditions, et puisque l'épaisseur de chaque couche mince a pour ordre de grandeur la longueur d'onde d'application, les composants IR seront approximativement 100 fois plus épais que les composants UV. Les problèmes de contraintes ou tenue mécaniques, dureté, d'adhérence..., s'en trouvent ainsi accrus et les solutions dépendent du domaine spectral. On retiendra également que la durée de fabrication est 100 fois plus importante pour les composants IR, ce qui pose un problème majeur et requiert une automatisation complète des systèmes de fabrication et contrôle.

\section{DISPERSION SPECTRALE DE LA RUGOSITE}

Pour la plupart des composants interférentiels, les pertes par diffusion lumineuse proviennent essentiellement des rugosités de surface. Les meilleurs polis optiques présentent souvent une rugosité aléatoire et à faible pente de l'ordre de l'Angström, mais il faut également considérer la présence de défauts localisés laissés par le polissage ou le nettoyage, qui contribuent aux taux de diffusion malgré leur faible densité. Pour d'autres substrats comme le Zéro-dur ou le $\mathrm{MgF}$, la diffusion volumique est prédominante et vient modifier les performances. Dans tous les cas, et compte tenu des périodes spatiales mises en jeu pour un bilan optique en champ lointain, on peut admettre que les empilements de couches minces viennent reproduire, couche après couche, et avec une faible altération, la rugosité du substrat, laquelle constitue ainsi un véritable seuil de diffusion. A cette rugosité "seuil » vient ensuite se superposer la rugosité intrinsèque aux matériaux et techniques utilisées pour le dépôt. Le résultat est une lumière diffuse issue des différentes interfaces, avec des degrés de cohérence mutuelle qui sont décrits à l'aide des coefficients d'intercorrélation entre interfaces. Cette cohérence partielle ou totale offre ainsi des solutions pour réduire la diffusion, ou pour l'exalter, selon que l'on veut scruter la surface ou le volume. 
Cette lumière diffuse est donc précieuse pour sonder la matière de façon non destructrice, et localiser les rugosités et hétérogénéités aux interfaces et volumes des composants. Toutefois la maîtrise de cette sonde suppose, lorsque l'on reçoit une lumière diffuse, que l'on soit capable d'en détecter l'origine (surface, volume, défauts discrets faiblement distribués ou rugosité dérivable...) sans ambiguïté. Des solutions directes ont été apportées grâce à des travaux sur les variations spectrales de la diffusion, ou sur la polarisation et l'anisotropie des flux mesurés. Plus récemment, on a pu montrer que l'ellipsométrie sur champ diffus constituait une technique idéale pour détecter l'origine de la diffusion.

Mais tout comme la notion de microstructure, la notion de rugosité n'a de sens que si l'on précise l'échelle d'observation, qui peut aller du macroscopique au microscopique. Pour notre application optique, et si l'on se limite à une étude en champ lointain, seules les périodes spatiales supérieures à la longueur d'onde contribuent aux pertes par diffusion, de sorte que la lumière ne verra pas les fréquences plus grandes que l'inverse de la longueur d'onde. On dit aussi abusivement que la lumière ne voit pas les défauts plus petits que la longueur d'onde. De façon plus précise, la bande passante accessible à l'expérience s'étale entre $\theta_{\mathrm{m}} / \lambda$ et $1 / \lambda$, avec $\lambda$ la longueur d'onde et $\theta_{\mathrm{m}}$ l'angle minimum de diffusion près du faisceau spéculaire : $\left.\mathrm{B}\left(\theta_{\mathrm{m}}, \lambda\right)=\right] \theta_{\mathrm{m}} / \lambda, 1 / \lambda[$

C'est dans cette bande passante que l'on mesure le spectre de rugosité des surfaces polies et composants, dont l'intégrale fréquentielle donne la rugosité recherchée. Cette rugosité est donc relative, et dépend de la longueur d'onde d'application. On montre que les spectres se superposent aux intersection des fenêtres fréquentielles, lorsque les longueurs d'onde varient de l'UV à l'IR. Dans ces conditions, on peut considérer que la rugosité aux très basses fréquences, responsables du flux diffusé dans le lointain IR, n'est autre que la planéité. A l'inverse, si l'on utilise l'imagerie obtenue par microscopie à force atomique, ou à sonde locale ou champ proche, les spectres et rugosités obtenus sont essentiellement microscopiques, dans une bande fréquentielle donnée par : $\left.B^{\prime}(L, \Delta x)=\right] 1 / L, 1 / \Delta x[$, où $L$ mesure le côté de la surface étudiée, et $\Delta x$ le pas d'échantillonnage.

On retiendra donc en général qu'une rugosité microscopique ne permet pas de prédire les pertes optiques par diffusion lumineuse, en dépit de la richesse des informations qu'elle transporte. De même, les rugosités macroscopiques (optiques) et microscopiques (AFM) sont égales si l'on impose la condition d'égalité entre la longueur d'onde et le pas d'échantillonnage : $\lambda \approx \Delta x$. Toutefois, cette condition n'est pas sans faille car les diamètres optiques utiles sont en général bien supérieurs aux régions étudiées par AFM, de sorte qu'il peut subsister des différences majeures entre rugosités microscopique et macroscopique, en raison de la présence de défauts localisés vus par la lumière et non par la pointe de l'outil en champ proche. Un compromis est donc en général nécessaire entre la résolution spatiale recherchée et l'extension de la zone étudiée, laquelle influe sur la résolution spectrale ou angulaire.

Ces considérations nous amènent immédiatement à adresser le problème de la dispersion spectrale de la rugosité. En d'autres termes, comment évolue la rugosité d'une surface si l'on fait croître la résolution de l'outil d'observation (effet de zoom)? Et puisque la résolution optique décroît avec la longueur d'onde, comment la rugosité et la diffusion évoluent-elles lorsque la longueur d'onde diminue? Un dioptre peut il être superpoli pour des applications visibles, et largement rugueux pour des longueurs d'onde X? Pour répondre à ces questions, on utilise le caractère linéaire de la rugosité en coordonnées bilogarithmiques, pour démontrer qu'il existe une opportunité de polissage multi-échelle lorsque la pente de la droite est maximale, ce qui correspond à la plus grande dimension fractale. Avec cette condition, les pertes par diffusion peuvent rester négligeable du visible aux rayons X. En l'absence de cette condition, la surface optique ne réfléchirait plus les ondes X....

\section{TENUE AU FLUX LASER}

Jusqu'ici les rugosités testées étaient essentiellement dérivables à faible pentes, ce qui laisse imaginer un profil de surface analogue à une fonction classiquement aléatoire. Nous avons également brièvement traité des défauts localisés faiblement đistribués, dont la présence se traduit par des oscillations dans le flux angulaire diffusé et mesuré à haute résolution (interférences de type Bessel bénéficiant d'une corrélation latérale sur l'échantillon). Nous discutons maintenant à nouveau de défauts isolés faiblement distribués ( 1 défaut tous les 100 $\mu \mathrm{m}$, pour une densité linéïque équivalente), mais non détectables par les techniques de diffusion lumineuse, en raison de leurs nano-diamètres pressentis. Ces défauts ou nanocentres jouent un rôle clé dans la physique de l'endommagement sous flux lumineux intense.

En effet, si l'on envoie, pour une énergie donnée, un pulse laser $(\Delta \mathrm{t}=7 \mathrm{~ns}, \lambda=1.06 \mu \mathrm{m})$ sur un échantillon, il arrive que la matière " casse » ou ne casse pas sous le flux incident. Ceci nous amène à effectuer une mesure statistique de l'endommagement sur $\mathrm{N}$ régions de l'échantillon. Pour chaque énergie $F$, on compte le nombre $p$ de zones qui ont «claqué », et le rapport $p / N$ donne une estimation de la probabilité d'endommagement. On constate alors qu'en dessous d'un 
seuil bas $F_{\min }$, aucune zone ne casse $(p=0)$, et qu'au dessus d'un seuil haut $F_{\text {haut, }}$, toutes les zones cassent $(p=1)$, mais qu'entre ces deux seuils apparaît une véritable courbe statistique. Celle-ci n'est pas très différente d'une estimation de la probabilité de présence de nanocentres sous la partie du faisceau laser où l'énergie est plus grande que le seuil de claquage du défaut $F_{\min }$. On notera que cette approche phénoménologique rend bien compte des processus de claquage, puisqu'elle permet de prévoir les seuils d'endommagement des solides et liquides, avec plusieurs types de défauts. Toutefois, elle n'explique pas la nature du phénomène d'endommagement (fusion, vaporisation, ablation, choc thermique, claquage diélectrique...).

De ces courbes de seuil nous sommes capables d'extraire la densité des nano-défauts initiateurs de l'endommagement, mais la méthode est ainsi destructrice et limite nos investigations. Quelles autres méthodes pouvons nous utiliser pour détecter ces nanocentres de façon non destructrice? Les mesures non optiques ne nous ont pas permis de conclure jusqu'ici, en raison de la faible densité des défauts (un nano-diamètre tous les $100 \mu \mathrm{m}$ ) qui les rend inaccessibles. Il en est de même pour les techniques de diffusion lumineuse ou de déflexion photothermique, dont les niveaux de détectivité dépendent du volume, choc d'indice et niveau d'absorption des impuretés... Actuellement ces techniques sont hybridées pour surmonter ces obstacles, et font appel à de la microscopie photothermique haute fréquence et haute résolution, ou à de la détection par diffusion en champ lointain de "boules " thermiques résultant de l'excitation de nanocentres...

\section{CONCLUSION}

On ne traite pas de la même façon les problèmes de filtrage optique UV ou IR, compte tenu des épaisseurs des composants et des temps de fabrication qui varient de plus d'un facteur $100 . \mathrm{La}$ même remarque s'applique vis-à-vis de la dimension des composants, qui varie de 6 décades selon l'application. De plus, la dispersion spectrale de la rugosité amène naturellement à rechercher des solutions multi-échelle, qu'explorera la lumière avec les différentes longueurs d'onde. Enfin, les défauts isolés très faiblement distribués avec des nanodiamètres demeurent pour l'instant inaccessibles avec les méthodes non destructrices, alors qu'ils constituent une clé pour la tenue au flux laser intense. Ces quelques considérations auront illustré succinctement, les notions de résolution spatiale, spectrale et fréquentielle, ou les niveaux de densité, qu'il faut reconsidérer selon l'échelle mise en jeu pour analyser les défauts dans les composants multicouches.

\section{Références}

1. C. Amra, Applied Optics, Vol.32, No28, pp. 5481-5491 (1993)

2. C. Amra, C. Grèzes-Besset, L. Bruel, Applied Optics, Vol. 32, N²8, pp. 5492-5503 (1993)

3. C. Amra, D. Torricini and P. Roche, Applied Optics, Vol. 32, pp. 5462-5474 (1993)

4. C. Amra, JOSA A, Vol. 11, N 1, pp. 197-210 and 211-226 (1994)

5. C. Deumié, R. Richier, P. Dumas and C. Amra, Applied Optics, Vol. 35, N²8, pp. 5583-5594 (1996)

6. S. Maure, G. Albrand and C. Amra, Applied Optics, Vol. 35, $\mathrm{N}^{\circ} 28$, pp. $5573-5582$ (1996)

7. C. Deumié, H. Giovannini and C. Amra, Applied Optics, Vol. 35, $\mathrm{N}^{\circ} 28$, pp 5600-5608 (1996)

8. H. Giovannini, C. Amra, Applied optics 36, N²2, pp 5574-5579 (1997)

9. H. Giovannini, C. Amra, Applied Optics, 37, 103-105 (1998) 